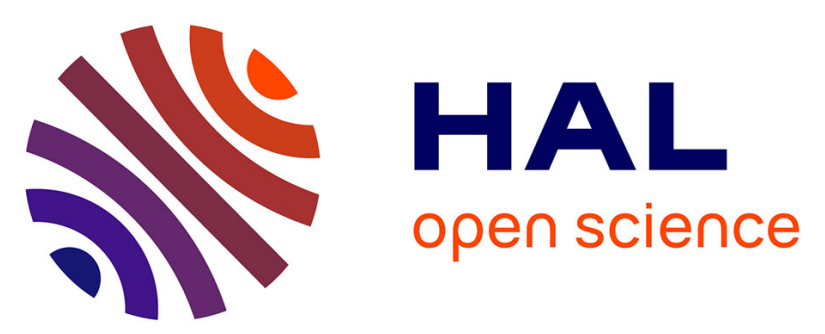

\title{
INTERACTION NON LINÉAIRE DE DEUX ONDES ACOUSTIQUES SUPERFICIELLES SE PROPAGEANT EN SENS OPPOSÉS A LA SURFACE D'UN CRISTAL PIÉZOÉLECTRIQUE
}

\author{
E. Bridoux, Jean-Michel Rouvaen, M. Delannoy, M. Moriamez
}

\section{To cite this version:}

E. Bridoux, Jean-Michel Rouvaen, M. Delannoy, M. Moriamez. INTERACTION NON LINÉAIRE DE DEUX ONDES ACOUSTIQUES SUPERFICIELLES SE PROPAGEANT EN SENS OPPOSÉS A LA SURFACE D'UN CRISTAL PIÉZOÉLECTRIQUE. Journal de Physique Colloques, 1972, 33 (C6), pp.C6-245-C6-251. 10.1051/jphyscol:1972653 . jpa-00215171

HAL Id: jpa-00215171

https://hal.science/jpa-00215171

Submitted on 1 Jan 1972

HAL is a multi-disciplinary open access archive for the deposit and dissemination of scientific research documents, whether they are published or not. The documents may come from teaching and research institutions in France or abroad, or from public or private research centers.
L'archive ouverte pluridisciplinaire HAL, est destinée au dépôt et à la diffusion de documents scientifiques de niveau recherche, publiés ou non, émanant des établissements d'enseignement et de recherche français ou étrangers, des laboratoires publics ou privés. 


\title{
INTERACTION NON LINÉAIRE DE DEUX ONDES ACOUSTIQUES SUPERFICIELLES SE PROPAGEANT EN SENS OPPOSÉS A LA SURFACE D'UN CRISTAL PIÉZOÉLECTRIQUE
}

\author{
E. BRIDOUX, J. M. ROUVAEN, M. DELANNOY et M. MORIAMEZ
}

\author{
Laboratoire d'Acoustique, Centre Universitaire, Mont Houy, 59 Valenciennes, France
}

\begin{abstract}
Résumé. - Un modèle unidimensionnel est proposé pour étudier l'interaction de deux ondes de Rayleigh de même fréquence se propageant en sens opposés à la surface d'un cristal piézoélectrique. La polarisation non linéaire induite à la surface du cristal par l'interaction des deux ondes est calculée en fonction d'une constante de couplage qui caractérise le milieu de propagation. Nous donnons les résultats expérimentaux obtenus à $150 \mathrm{MHz}$ avec des ondes de Rayleigh se propageant suivant l'axe $Z$ d'un cristal de niobate de lithium de coupe $Y$. L'enveloppe du signal hyperfréquence détecté à la fréquence double représente la convolution des signaux de modulation des deux ondes acoustiques qui interagissent.
\end{abstract}

\begin{abstract}
A one dimensional model is proposed for studying the interaction between two Rayleigh electroacoustic surface waves of the same frequency and opposite propagation directions on a piezoelectric crystal. The nonlinear polarization induced on the crystal's surface via the two interacting waves is obtained in terms of a nonlinear coupling constant characterizing the propagation medium. We report some experimental results for $150 \mathrm{MHz}, \mathrm{Z}$ propagating, Rayleigh waves on $\mathrm{Y}$ cut lithium niobate crystal. The amplitude modulation of the radiofrequency signal detected at twice the inputfrequency represents the convolution product of the individual amplitude modulations of the two interacting electroacoustic waves.
\end{abstract}

1. Introduction. - Dans les solides cristallins, plusieurs sortes d'interactions non linéaires sont possibles entre ondes acoustiques de surface. Certaines d'entre elles font principalement intervenir les propriétés élastiques du cristal : la génération des ondes acoustiques de fréquences harmoniques, celle des ondes aux fréquences somme et différence ainsi que l'amplification paramétrique sont alors dues aux dislocations [1] ou aux anharmonicités des forces interatomiques [2]. Plusieurs études ont été effectuées sur ces effets dans le niobate de lithium [3], [4], [5].

Un autre type d'interaction faisant intervenir la piézoélectricité du cristal a été étudié par L. O. Svaasand [6] dans le quartz, M. V. Luukkala et G. S. Kino [7] dans le niobate de lithium et E. A. Kraut, T. C. Lim et B. R. Tittmann [8] dans les céramiques piézoélectriques. Cette interaction non linéaire est à l'origine de la génération à partir d'ondes de surface de pulsation et de vecteurs d'onde respectifs $\left(\omega_{1}, k_{1}\right)$ et $\left(\omega_{2}, k_{2}\right)$ d'un champ électrique de pulsation $\left(\omega_{1} \pm \omega_{2}\right)$ et de vecteur d'onde $\left(k_{1} \pm k_{2}\right)$. Plusieurs composants acoustiques destinés au traitement des signaux sont basés sur cet effet [7], [9], [10], [11].

Nous proposons ici un modèle monodimensionnel permettant de décrire ce processus, appelé convolution acoustique en surface, au moyen d'un paramètre non linéaire unique. La validité de ce modèle est testée en comparant la valeur de ce paramètre unique avec celui introduit par J. D. Larson [12] pour décrire la convolution acoustique en volume dans le niobate de lithium.

2. Convolution acoustique en surface. - Considérons deux ondes acoustiques de surface, se propageant en sens opposés sur la face d'un cristal de niobate de lithium, dont les composantes varient respectivement en :

$$
\exp i\left(\omega_{1} t-k_{1} z\right) \text { et } \exp i\left(\omega_{2} t+k_{2} z\right)
$$

où $z$ représente la direction de propagation.

La composante de déformation mécanique produite par interaction non linéaire varie comme le produit des deux signaux incidents, soit en :

$$
\exp i\left[\left(\omega_{1}+\omega_{2}\right) t-\left(k_{1}-k_{2}\right) z\right] \text {. }
$$

Un champ électrique possédant les mêmes variations en fonction du temps et de la coordonnée spatiale $z$ est associé à la déformation précédente.

Dans le cas particulier où $\omega_{1}=\omega_{2}=\omega$ et $k_{1}=k_{2}=k$, le champ électrique produit est uniforme dans l'espace et varie dans le temps avec la pulsation $2 \omega$. Ceci a été montré expérimentalement par L. O. Svaasand [6] dont le circuit détecteur consistait en deux couches métalliques parallèles déposées sur les deux faces d'une ligne à ondes de surface. Plus récemment, C. F. Quate et R. B. Thompson [13] ont montré que, lorsque les deux signaux d'entrée sont modulés, la modulation du signal de sortie est le produit de convolution acoustique des modulations 
des signaux d'entrée. Cette application importante des interactions non linéaires, qu'ils ont démontrée expérimentalement en utilisant des ondes acoustiques de volume, a été vérifiée pour les ondes acoustiques de surface par M. Luukkala et G. S. Kino [7].

Si nous considérons l'interaction de deux signaux modulés (Fig. 1)

$$
F(t) \exp i\left(\omega_{1} t-k_{1} z\right) \text { et } G(t) \exp i\left(\omega_{2} t+k_{2} z\right)
$$

se propageant en sens inverse sur la surface d'un cristal, le signal produit paramétriquement est de la forme :

$C \cdot F\left(t-\frac{z}{v}\right) G\left(t+\frac{z}{v}\right) \exp i\left[\left(\omega_{1}+\omega_{2}\right) t-\left(k_{1}-k_{2}\right) z\right]$

où $v$ est la vitesse des ondes acoustiques et $\mathrm{C}$ un paramètre de couplage. Le circuit détecteur capte ce signal sur une longueur $L$ égale à celle des électrodes, de sorte que la modulation du signal disponible à la sortie peut s'écrire :

$$
H(t)=\mathrm{C} \int_{-L / 2}^{L / 2} F\left(t-\frac{z}{v}\right) G\left(t+\frac{z}{v}\right) \mathrm{d} z
$$

Lorsque les signaux d'entrée sont des impulsions de largeur très inférieure au temps de transit des ondes acoustiques sous les électrodes détectrices, la longueur $L$ de la région détectrice peut être consi- dérée comme infinie. En effectuant alors le changement de variable $\tau=t-(z / v)$, il vient :

$$
H(t)=-C v \int_{-\infty}^{+\infty} F(\tau) G(2 t-\tau) \mathrm{d} \tau .
$$

La modulation du signal de sortie peut être considérée comme le produit de convolution des modulations des signaux d'entrée au facteur 2 près, le produit de convolution mathématique exact étant:

$$
h(t)=\int_{-\infty}^{+\infty} F(\tau) G(t-\tau) \mathrm{d} \tau .
$$

La convolution acoustique représentée par $H(t)$ peut alors être reliée à la convolution mathématique :

$$
H(t)=-\operatorname{Cvh}(2 t) .
$$

2.1 ETUDE DE LA CONVOLUTION ACOUSTIQUe A PARTIR DE L'ÉNERGIE LIBRE NON LINÉAIRE. - Afin de rendre compte des propriétés non linéaires du cristal, nous pouvons écrire l'énergie libre isentropique [14] en nous limitant aux termes du troisième ordre :

$$
\begin{aligned}
U= & -\frac{1}{2} c_{i j k l}^{E} S_{i j} S_{k l}-\frac{1}{6} c_{i j k l m n}^{E} S_{i j} S_{k l} S_{m n} \\
& +\frac{1}{2} \varepsilon_{i j}^{S} E_{i} E_{j}+\frac{1}{6} g_{i j k}^{S} E_{i} E_{j} E_{k}+e_{i j k} E_{i} S_{j k} \\
& +\frac{1}{2} d_{i j k l} E_{i} E_{j} S_{k l}+\frac{1}{2} f_{i j k l m} E_{i} S_{j k} S_{l m}
\end{aligned}
$$

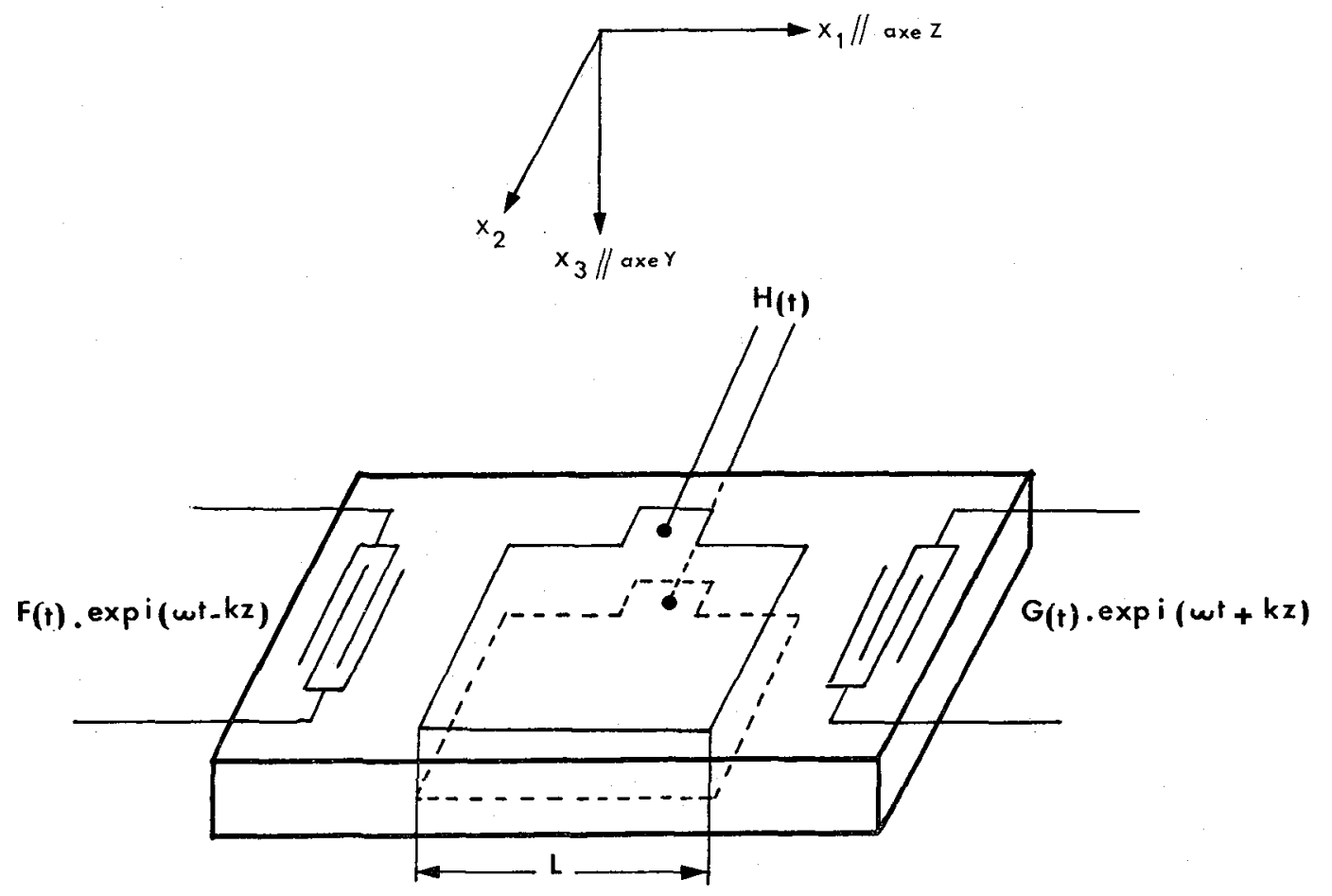

$$
H(t)=C \int_{-\frac{L}{2}}^{\frac{L}{2}} F\left(t-\frac{z}{v}\right) \cdot G\left(t+\frac{z}{v}\right) d z
$$

FIG. 1. - Principe de la convolution acoustique. 
où les $S_{j k}$ sont les composantes de la déformation mécanique et $E_{i}$ celles du champ électrique.

Les constantes introduites dans cette relation sont explicitées ci-dessous :

$-c_{i j k l}^{E}$ et $c_{i j k l m n}^{E}$ sont les constantes élastiques $\mathrm{du}$ second ordre et du troisième ordre à champ électrique constant.

$-\varepsilon_{i j}^{S}$ et $g_{i j k}^{S}$ sont les constantes de permittivité électrique du second ordre et du troisième ordre à déformation mécanique constante.

- $e_{i j k}$ sont les constantes piézoélectriques.

- $d_{i j k l}$ sont les constantes électrostrictives.

- $f_{i j k l m}$ sont les constantes électroacoustiques.

A partir de cette expression de l'énergie libre, nous pouvons obtenir la tension mécanique dans le cristal et le déplacement électrique induit en fonction de la déformation mécanique et du champ électrique :

$$
\begin{aligned}
T_{i j}= & -\frac{\partial U}{\partial S_{i j}} \\
=c_{i j k l}^{E} S_{k l} & +\frac{1}{2} c_{i j k l m n}^{E} S_{k l} S_{m n}-e_{k i j} E_{k}- \\
& \quad-\frac{1}{2} d_{k l i j} E_{k} E_{l}-f_{m i j k l} E_{m} S_{k l} \\
D_{i}= & \frac{\partial U}{\partial E_{i}}= \\
= & \varepsilon_{i j}^{S} E_{j}+\mathrm{e}_{i j k} S_{j k}+\frac{1}{2} g_{i j k}^{S} E_{j} E_{k}+ \\
& \quad+d_{i j k l} E_{j} S_{k l}+\frac{1}{2} f_{i j k l m} S_{j k} S_{l m} .
\end{aligned}
$$

La propagation des ondes acoustiques superficielles peut être étudiée en utilisant les équations du mouvement :

$$
\rho \frac{\partial^{2} u_{j}}{\partial t^{2}}=\frac{\partial T_{i j}}{\partial x_{i}}
$$

et la loi de Gauss en supposant le cristal isolant :

$$
\frac{\partial D_{i}}{\partial x_{i}}=0
$$

Les ondes acoustiques superficielles sont bidimensionnelles et non homogènes. Les équations précédentes sont donc excessivement compliquées dans le cas d'un cristal anisotrope comme le niobate de lithium et leur résolution est pratiquement impossible car la plupart des constantes du troisième ordre n'ont encore pu être mesurées pour ce cristal.

Aussi utiliserons-nous, comme pour traiter le cas de la génération harmonique [15], un modèle monodimensionnel faisant appel à un paramètre non linéaire unique pour calculer la polarisation non linéaire responsable de la convolution acoustique.

2.2 Modèle MONOdIMENSIONNEL. - Des programmes de calcul numérique permettent de relier la composante normale du déplacement mécanique $u_{3}$ à la surface du cristal et la puissance acoustique par unité de largeur du transducteur [16]

$$
\left[u_{3}\right]^{2}=M \frac{P_{\mathrm{ac}}}{f d}
$$

où $f$ est la fréquence de l'onde acoustique, $M$ une constante dépendant des propriétés du cristal et $d$ la largeur du transducteur.

La puissance acoustique $P_{\mathrm{ac}}$ peut être calculée à partir du vecteur de Poynting acoustique. Considérons une onde acoustique de surface se propageant selon la direction $x_{1}$ comme l'indique la figure 1 .

Le vecteur de Poynting acoustique a pour composante dans la direction de propagation :

$$
P_{1}=-\frac{1}{2}\left(T_{11} \frac{\partial u_{1}^{*}}{\partial t}+T_{13} \frac{\partial u_{3}^{*}}{\partial t}\right) \text {. }
$$

La puissance acoustique par unité de largeur du transducteur s'obtient en intégrant cette quantité sur la profondeur du cristal dans un plan normal à la direction de propagation $x_{1}$ :

$$
\frac{P_{\mathrm{ac}}}{d}=\int_{0}^{\infty} P_{1} \mathrm{~d} x_{3} .
$$

Comme dans le cas de la génération harmonique [15], nous pouvons choisir la phase de référence de telle façon que les composantes normales des ondes fondamentales s'écrivent :

$$
\begin{aligned}
& u_{+}=A\left(t-\frac{z}{v}\right) \cos (\omega t-k z) \exp \left[-\alpha\left(\frac{L}{2}+z\right)\right] \\
& u_{-}=B\left(t+\frac{z}{v}\right) \cos (\omega t+k z) \exp \left[-\alpha\left(\frac{L}{2}-z\right)\right]
\end{aligned}
$$

où $\alpha$ représente l'atténuation des ondes acoustiques de surface lors de la propagation sous les électrodes détectrices et $A$ et $B$ sont des amplitudes réelles qui d'après (2) peuvent s'écrire :

$$
\begin{aligned}
& A=\sqrt{\frac{M P_{+}}{f d}} f\left(t-\frac{z}{v}\right) \\
& B=\sqrt{\frac{M P_{-}}{f d}} g\left(t+\frac{z}{v}\right)
\end{aligned}
$$

où $P_{+}$et $P_{-}$sont les puissances acoustiques transportées par les ondes acoustiques incidentes et $f$ et $g$ sont les modulations de ces deux ondes. Le déplacement mécanique total s'écrit :

$$
\begin{gathered}
u=u_{+}+u_{-}=\sqrt{\frac{M}{f d}} \times \\
\times\left[\sqrt{P_{+}} f\left(t-\frac{z}{v}\right) \cos (\omega t-k z) \exp \left[-\alpha\left(\frac{L}{2}+z\right)\right]\right. \\
+\sqrt{P_{-}} g\left(t+\frac{z}{v}\right) \cos (\omega t+k z) \exp \left[-\alpha\left(\frac{L}{2}-z\right)\right] .
\end{gathered}
$$


Le déplacement électrique induit (1) s'écrit dans l'approximation monodimensionnelle

$$
D=D_{\mathrm{L}}+D_{\mathrm{NL}}
$$

avec :

$$
\begin{aligned}
& D_{\mathrm{L}}=\varepsilon E+e \frac{\partial u}{\partial z} \\
& D_{\mathrm{NL}}=\frac{1}{2} g E^{2}+\mathrm{d} E \frac{\partial u}{\partial z}+\frac{1}{2} f\left(\frac{\partial u}{\partial z}\right)^{2} .
\end{aligned}
$$

Le terme linéaire permet d'évaluer le champ électrique associé aux ondes acoustiques :

$$
E=-\frac{e}{\varepsilon} \frac{\partial u}{\partial z}
$$

en supposant $D_{\mathrm{L}}=0$.

Cela permet de calculer la polarisation non linéaire qui s'écrit :

$$
D_{\mathrm{NL}}=K\left(\frac{\partial u}{\partial z}\right)^{2}
$$

avec

$$
K=\frac{1}{2} g \frac{e^{2}}{\varepsilon^{2}}-d \frac{e}{\varepsilon}+\frac{1}{2} f .
$$

Le résultat exprimé par la relation (4) montre que le type d'interaction envisagé n'est possible que si le cristal est piézoélectrique et que la polarisation non linéaire $D_{\mathrm{NL}}$ est proportionnelle au carré de la déformation mécanique.

L'amplitude du terme non linéaire variant avec la pulsation $2 \omega$ peut s'exprimer, en reportant le déplacement mécanique (3) dans l'éq. (4), comme :

$\left|D_{\mathrm{NL}}\right|=K \frac{k^{2} M}{f d} \sqrt{P_{+} P_{-}} f\left(t-\frac{z}{v}\right) g\left(t+\frac{z}{v}\right) e^{-\alpha L}$.

2.3 CircutT ÉleCtrique ÉQuivalent Au CRISTAL. Nous pouvons supposer la polarisation non linéaire $D_{\mathrm{NL}}$ constante à l'intérieur du cristal sur une couche superficielle épaisse d'une longueur d'onde acoustique (ce qui correspond approximativement à la distance de pénétration des ondes acoustiques superficielles dans le niobate [17]) et nulle à des profondeurs supérieures.

La zone détectrice se comporte donc comme un générateur de tension $\lambda / \varepsilon \quad D_{\mathrm{NL}}$ dont l'impédance interne est constituée par la capacité équivalente au cristal $\varepsilon A / h$ en parallèle avec une résistance de fuite $R$ où $h$ représente l'épaisseur du cristal et $A$ la surface d'une électrode détectrice (Fig. 2). La résistance $R \mathrm{du}$ cristal isolant est supérieure de plusieurs ordres de grandeur à l'impédance de charge $Z_{0}(=50 \Omega)$ et peut être négligée.

Le circuit électrique équivalent au cristal représenté à la figure 2 permet de calculer la tension

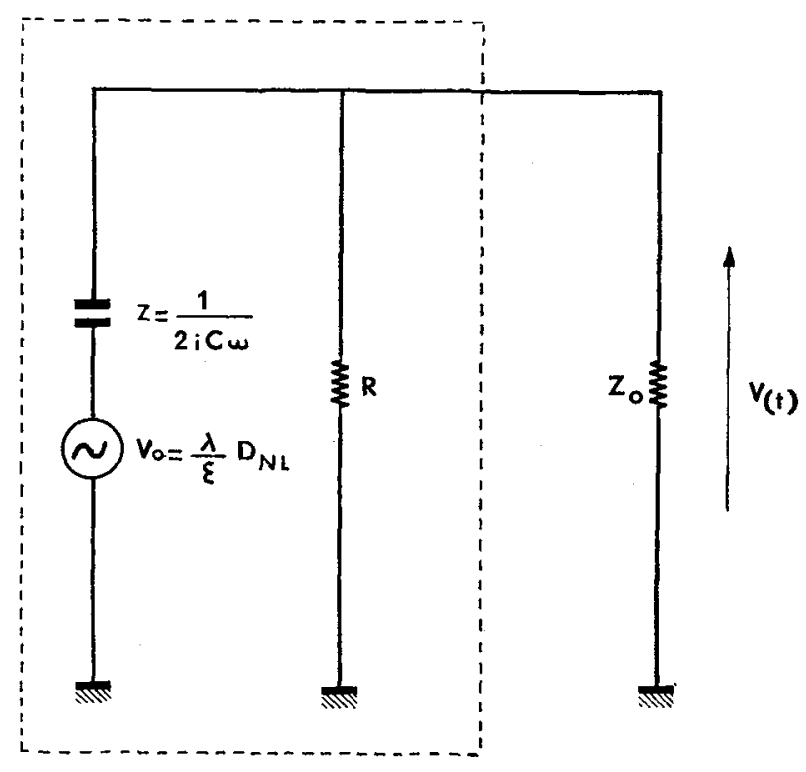

FIG. 2. - Circuit électrique équivalent.

$V(t)$ de convolution recueillie aux bornes de l'impédance de charge $Z_{0}$ :

$$
V(t)=\frac{Z_{0}}{Z_{0}+Z} \frac{\lambda}{\varepsilon} \frac{1}{L} \int_{-L / 2}^{L / 2} D_{\mathrm{NL}} \mathrm{d} z
$$

en supposant la largeur de l'électrode détectrice analogue à celle du transducteur.

Si les deux signaux d'entrée sont de durée très inférieure au temps de transit des ondes acoustiques sous les électrodes détectrices, le changement de variable $\tau=t-z / v$ permet d'écrire, en utilisant (5) :

$$
\begin{array}{r}
V(t)=-\frac{Z_{0}}{Z_{0}+Z} \frac{4 \pi^{2} M \mathrm{e}^{-\alpha L}}{\varepsilon L} K \frac{\sqrt{P_{+} P_{-}}}{d} \times \\
\times \int_{-\infty}^{+\infty} f(\tau) g(2 t-\tau) \mathrm{d} \tau .
\end{array}
$$

Considérons le cas de deux impulsions acoustiques rectangulaires d'entrée de même durée $\tau_{s}$ et de puissances égales $P_{+}=P_{-}=P_{\mathrm{ac}}$.

Alors nous avons :

$$
f(t), g(t)=\left\{\begin{array}{lll}
1 & \text { si } & 0 \leqslant t \leqslant \tau_{\mathrm{s}} \\
0 & \text { si } & t>\tau_{\mathrm{s}}
\end{array}\right.
$$

et nous voyons aisément que :

$$
\int_{-\infty}^{+\infty} f(\tau) g(2 t-\tau) \mathrm{d} \tau= \begin{cases}2\left(\tau_{\mathrm{s}}-t\right) & \text { si } t>\tau_{\mathrm{s}} / 2 \\ 2 t & \text { si } t \leqslant \tau_{\mathrm{s}} / 2 .\end{cases}
$$

La convolution acoustique de deux impulsions rectangulaires donne donc une impulsion triangulaire et la tension de convolution maximale, recueillie au temps $t=\tau_{\mathrm{s}} / 2$, est d'après (6) :

$$
V_{\max }=\left|\frac{Z_{0}}{Z_{0}+Z}\right| \frac{4 \pi^{2 \frac{2}{2}} M \mathrm{e}^{-\alpha L}}{\varepsilon L} K \frac{P_{\mathrm{ac}}}{d} \tau_{\mathrm{s}} .
$$



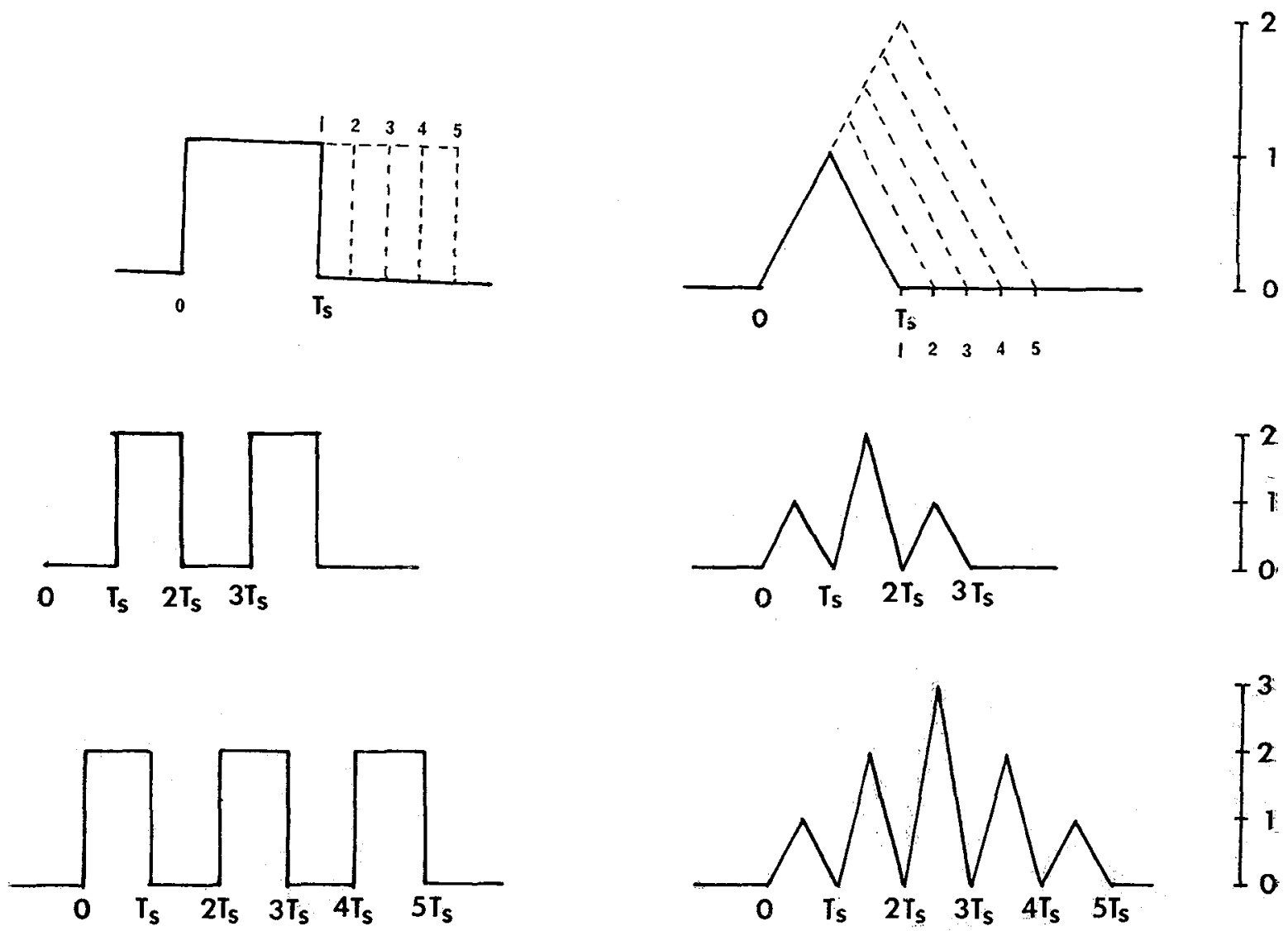

Modulation du signal incident

\section{Convolution acoustique}

(unites arbitraires)

FIG. 3. - Convolution acoustique d'impulsions HF rectangulaires.

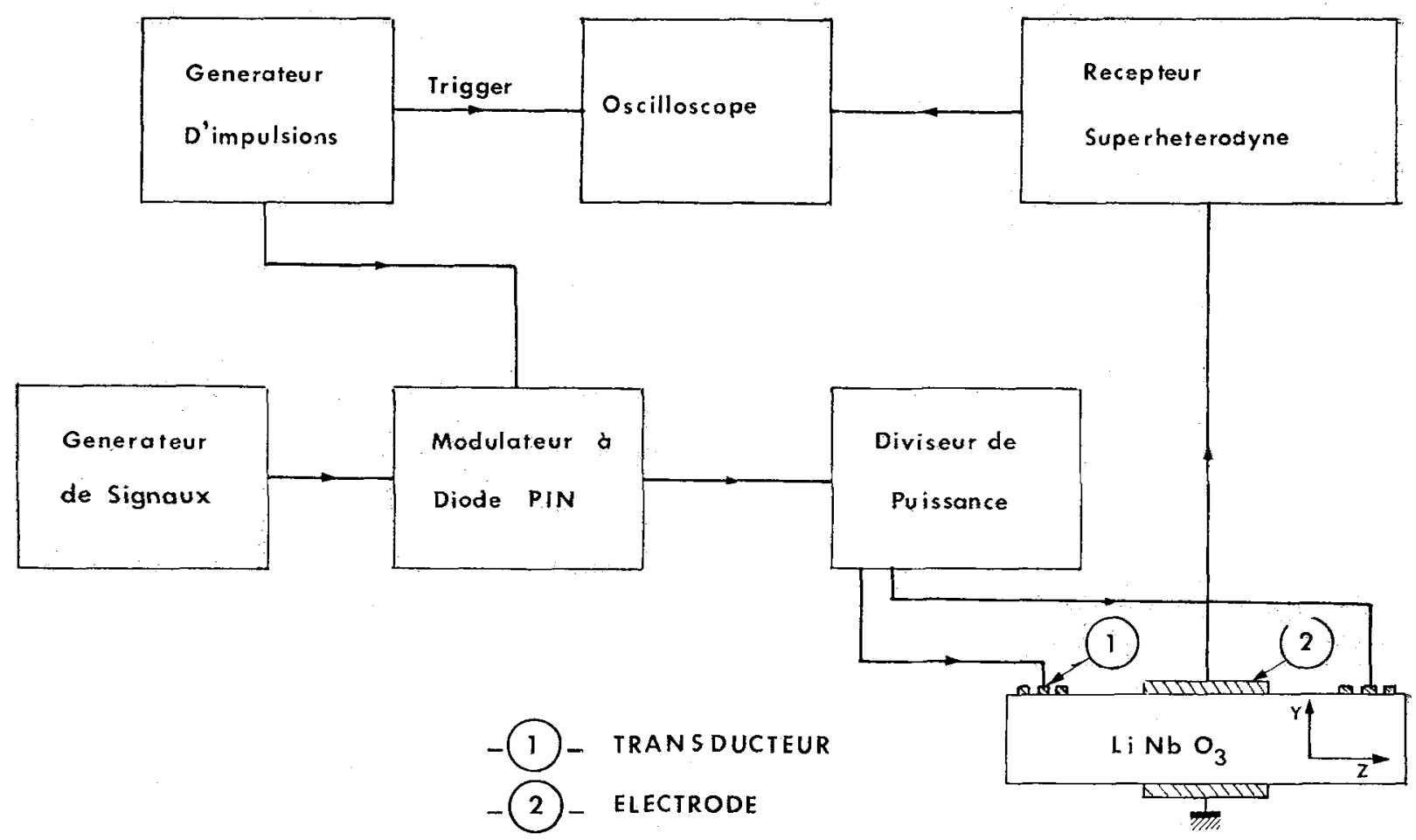

FIG. 4. - Montage expérimental. 
La tension maximale de convolution (7) est donc proportionnelle à la durée de l'impulsion $\tau_{\mathrm{s}}$ et à la puissance acoustique par unité de largeur du transducteur $P_{\mathrm{ac}} / d$, contrairement au cas de la génération harmonique où la tension maximale est proportionnelle à la densité de puissance acoustique $P_{\text {ac }} / \lambda . d[15]$.

La figure 3 représente la convolution acoustique de divers signaux simples.

2.4 RÉSUltatS EXPÉRIMENTAUX. - Le cristal utilisé lors des expériences était une plaquette de niobate de lithium de coupe $\mathrm{Y}$ d'épaisseur égale à $2 \mathrm{~mm}$, la propagation s'effectuant selon l'axe Z. Sur la surface de ce cristal, deux transducteurs interdigités identiques de largeur $2 \mathrm{~mm}$ ont été fabriqués par gravure d'un film d'aluminium d'épaisseur $4000 \AA$ de même qu'une électrode détectrice longue de $20 \mathrm{~mm}$ et large de
$2 \mathrm{~mm}$. Sur l'autre face du cristal une électrode détectrice identique à la précédente et placée en regard a été également fabriquée.

La perte d'insertion électrique, mesurée à $150 \mathrm{MHz}$ entre les deux transducteurs, était de $20 \mathrm{~dB}$. L'étude effectuée sur la ligne au moyen d'une sonde optique a permis de montrer que l'électrode métallique déposée sur la surface de propagation introduisait une perte de $10 \mathrm{~dB}$. Pour cette dernière mesure nous avons utilisé la diffraction d'un faisceau laser de longueur d'onde $6328 \AA$ par réflexion due à la déformation de la surface liée au passage de l'onde acoustique [18]. La convolution acoustique a été ensuite étudiée au moyen du montage expérimental de la figure 4.

Nous avons obtenu les deux signaux d'entrée à partir du même générateur en utilisant un diviseur de puissance. De cette façon, nous avons envoyé sur chaque transducteur des impulsions de durée
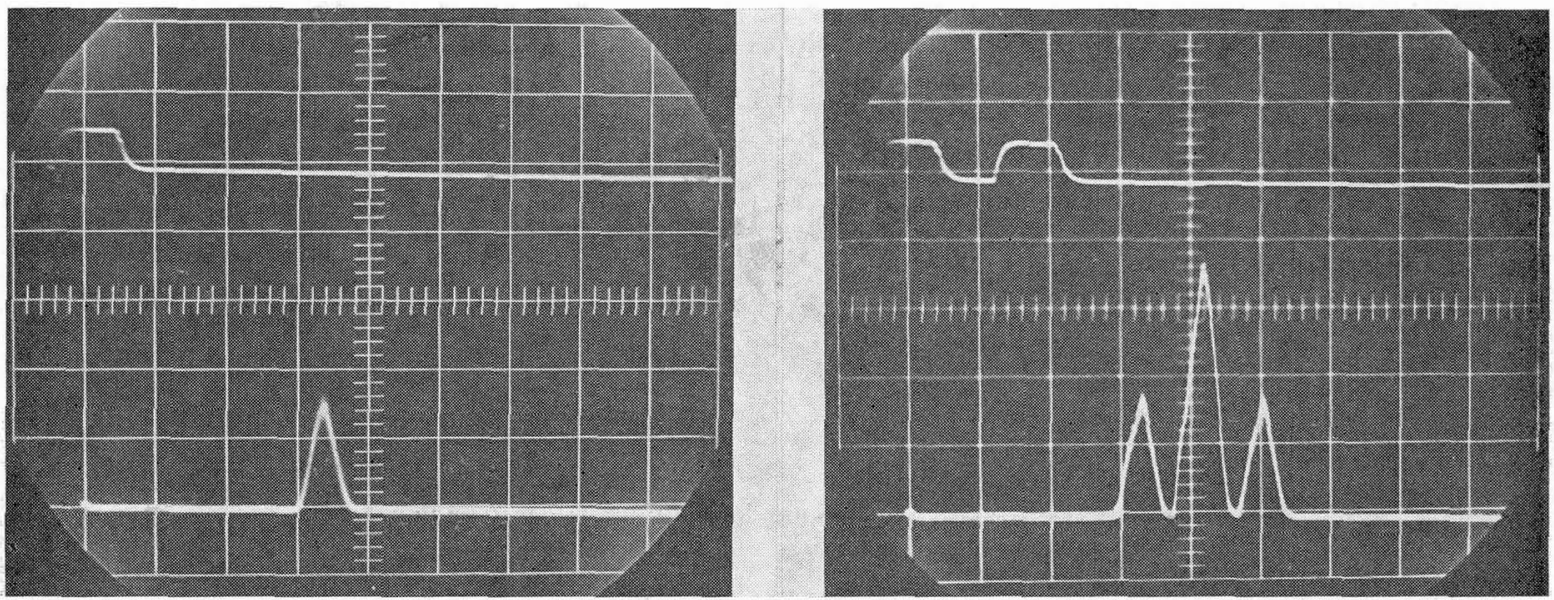

(a)
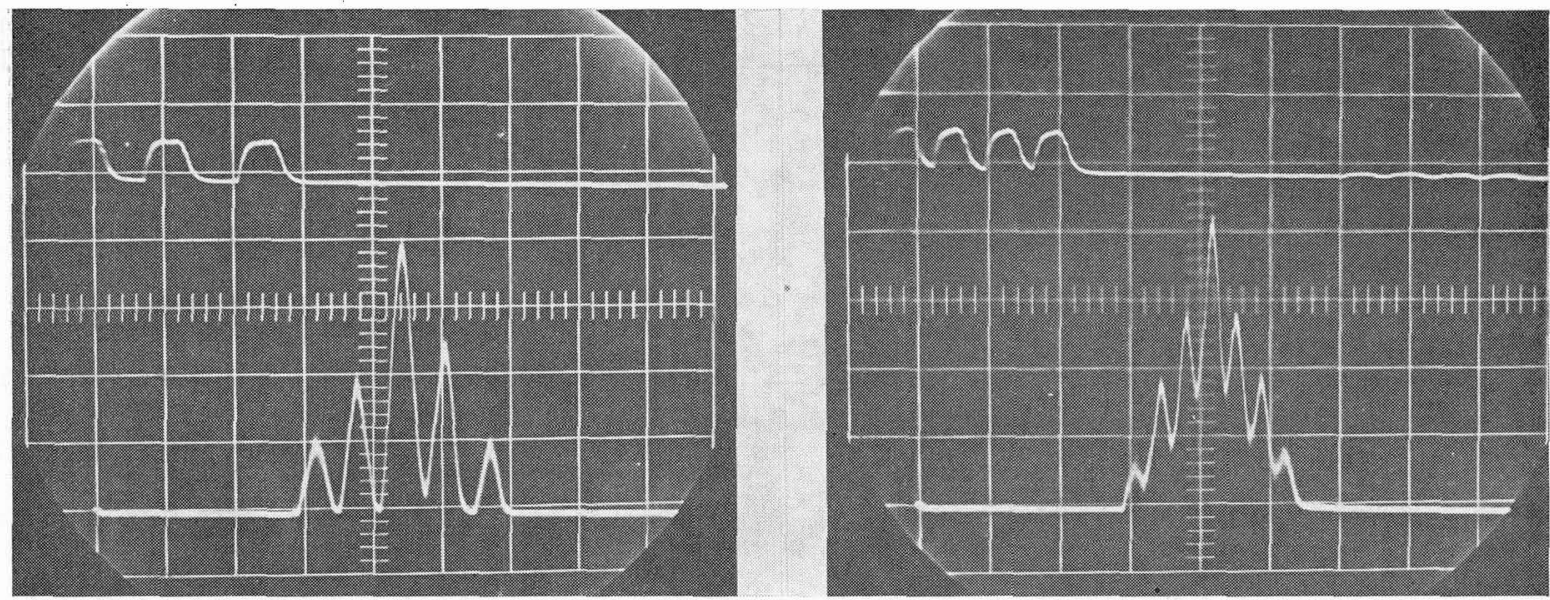

(b)

FIG. 5. - Résultats expérimentaux.

- La trace supérieure représente les impulsions rectangulaires incidentes au nombre de 1, 2, 3 et 4.

- La trace inférieure représente l'auto-convolution des signaux incidents (échelle horizontale ; $2 \mu$ sar division). 
égale et de puissance égale. Nous avons vérifié que la convolution acoustique des deux impulsions rectangulaires est une impuision triangulaire et que la tension maximale de convolution est proportionnelle à la durée des impulsions. L'éq. (7) a alors pu être utilisée pour calculer le paramètre non linéaire unique $K$ décrivant la convolution acoustique grâce aux résultats suivants : pour des impulsions de durée $6 \mu$ s et de puissance crête $1 \mathrm{~W}$, la tension maximale de convolution était de $3 \mathrm{mV}$. La valeur de la constante $M$, déterminée à partir de programmes de calcul numérique, est dans le cas d'un cristal de niobate de lithium de coupe $Y$, la propagation s'effectuant selon l'axe $Z$ de $2 \times 10^{-12}$. Nous obtenons alors: $K=8 \pm 3$ coulombs $\mathrm{m}^{-2}$.

L'incertitude expérimentale sur la perte de propagation sous l'électrode métallique est la principale source d'erreur pour le calcul de $K$.

Nous avons en outre vérifié la proportionnalité entre la tension maximale de convolution et la racine carrée de la puissance d'un des deux signaux acoustiques incidents.

De plus les cas envisagés à la figure 3 ont été vérifiés expérimentalement comme le montrent les photographies de la figure 5.

3. Conclusion. - Récemment, nous avons également utilisé un modèle monodimensionnel faisant appel à un paramètre unique $\beta$ pour décrire la génération d'ondes acoustiques de surface aux fréquences harmoniques [15]. Ce type d'interaction non linéaire fait intervenir à la fois les propriétés élastiques et électriques du cristal. Dans ce travail antérieur, une valeur de 0,53 a été trouvée pour le paramètre $\beta$ dans le cas d'un cristal de niobate de lithium de coupe $Y$ et de direction de propagation $Z$.

En utilisant l'approximation monodimensionnelle, nous voyons que :

$$
\beta=\frac{\frac{1}{2} c_{111}-\frac{1}{2} d \frac{e^{2}}{\varepsilon^{2}}+f \frac{e}{\varepsilon}}{c_{11}+\frac{e^{2}}{\varepsilon}}
$$

en désignant par $c_{11}$ et $c_{111}$ les constantes élastiques monodimensionnelles du second ordre et du troisième ordre respectivement. L'expression du paramètre $K$ définie à l'éq. (4) fait intervenir des combinaisons différentes de constantes du troisième ordre, ce qui rend toute comparaison avec le paramètre $\beta$ très difficile.

Cependant la valeur de $K=8$ coulombs $\mathrm{m}^{-2}$ trouvée pour la convolution acoustique en surface est du même ordre de grandeur que les 4,8 coulombs $\mathrm{m}^{-2}$ donnés par Larson [12] pour la convolution acoustique en volume dans le niobate de lithium selon l'axe cristallographique $X$. Cela semble néanmoins indiquer une sensibilité supérieure pour la convolution acoustique en surface. Mais comme la puissance maximale supportable est beaucoup plus faible pour une ligne à ondes de surface que pour une ligne à ondes de volume, la tension maximale de convolution étant proportionnelle d'après (7) à la puissance acoustique $P_{\text {ac }}$, le dispositif à ondes de surface ne présente pas d'avantage par rapport au dispositif à ondes de volume (contrairement au cas de la génération harmonique).

En conclusion nous avons étudié expérimentalement l'interaction non linéaire de deux ondes acoustiques superficielles se propageant en sens opposés sur la surface d'un cristal piézoélectrique. Cette interaction possède une application importante, la convolution acoustique en surface, qui a été décrite au moyen d'un modèle monodimensionnel faisant intervenir un paramètre non linéaire unique $K$. Les résultats expérimentaux ont permis de trouver pour ce paramètre une valeur voisine de celles des paramètres correspondants en ondes de volume.

\section{Bibliographie}

[1] Rischiteter F., Acoustica 18 (1967) 109.

[2] Lopen P. O., J. Appl. Phys. 39 (1968) 5400.

[3] Lean E. G., Tseng C. C., et Powell C. G., Appl. Phys. Lett 15 (1970) 32.

[4] Lean E. G. et Tseng C. C., J. Appl. Phys. 41 (1970) 3912.

[5] Slobodnik A. J., J. Acoust. Soc. Am. 48 (1970) 203.

[6] SvaAsand L. O., Appl. Phys. Lett 15 (1969) 300.

[7] Luukkala M. et Kino G. S., Appl. Phys. Lett 18 (1971) 393.

[8] Kraut E. A., Lim T. C. et Tittmann B. R., IEEE Symposium on Applications of Ferroelectricity (1971) à paraître.

[9] Bongtanni W. L., Proc. IEEE 59 (1971) 713.

[10] Shreve W. R., Kino G. S. et LuUkKala M., Electron. Lett. 7 (1971) 764.
[11] Morgan D. P., Darby B. J. et Collins J. H., Electron. Lett 8 (1972) 40.

[12] Larson J. D., Ph. D. Dissertation, Microwave Laboratory, Stanford University, Stanford, Calif. (1970).

[13] Quate C. F. et Thompson R. B., Appl. Phys. Lett 16 (1970) 494.

[14] McMahon D. H., J. Acoust. Soc. Am. 44 (1968) 1007.

[15] Adler E. L., Bridoux E., Coussot G. et DieuleSAINX E., IEEE, Trans. Sonics and Ultrasonics, à paraître.

[16] WhITe R. M., IEEE, Trans. Electron Devices, 1967, ED $14,181$.

[17] Moriamez M., Bridoux E., Desrumaux J. M., Rouvaen J. M. et Delannoy M., Revue Phys. Appl. 6 (1971) 333.

[18] Lean E. G. et Powell C. G., Proc. IEeE 58 (1970) 1939. 